haben, anders als bei Haushaltsabfällen, keine Möglichkeit, einen Anschluss- und Benutzungszwang mit Gebührenfinanzierung durchzusetzen.

Zumindest ein Teil der Schwierigkeiten und Risiken könnte vermieden werden, wenn die unterschiedlichen bei Auffüllungen und Aufschüttungen zur Anwendung kommenden Bundes- und Landesgesetze aufeinander abgestimmt wären und die zuständigen Behörden bundesweit nach einheitlichen Richtlinien vorgehen würden. Dies würde jedenfalls zu besserer Planbarkeit und Kostentransparenz von Bau- und Entsorgungsmaßnahmen beitragen.
Des Weiteren braucht es ein stabiles und dauerhaftes „Bündnis für Kreislaufwirtschaft auf dem Bau“" ${ }^{33}$, das heißt Zusammenarbeit zwischen der öffentlichen und der privaten Bauwirtschaft und ebenso der öffentlichen und der privaten Entsorgungswirtschaft. Die gemeinsame Nutzung von Know How und Entsorgungskapazitäten wird für alle Beteiligten von Vorteil sein.

33) So der Titel eines Beitrags von Nonte in Müll und Abfall 2014, $532-535$.

\title{
BUCHBESPRECHUNGEN
}

DOI: 10.1007/s10357-015-2822-9

\section{Oliver Ruppel/Christian Roschmann/Katharina Ruppel-Schlichting (Hrsg.): Climate Change: International Law and Global Governance}

Volume I: Legal Responses and Global Responsibility, 2013, 1016 S., ISBN 978-3-8329-7797-9, 168,00 Euro

Volume II: Policy, Diplomacy and Governance in a Changing Environment, 2013, 926 S., ISBN 978-3-8329-7796-2, 168,00 Euro, Nomos Verlagsgesellschaft, Baden-Baden

Der fortschreitende Klimawandel führt zu weitreichenden Veränderungen der Umwelt. Die Grenzen der Verbreitungsgebiete vieler Arten verschieben sich. Arten mit höheren Temperaturansprüchen (submediterrane, mediterrane und atlantische Arten, aber auch subtropische und tropische Neobiota) dehnen bereits heute ihr Areal horizontal nach Norden und Osten bzw. im Gebirge vertikal um einige hundert Höhenmeter nach oben aus. Auch Vögel reagieren auf vielfältige Weise auf den Klimawandel, so z.B. durch die Vorverlegung des Brutbeginns bei Temperaturanstieg im Frühjahr, Änderungen von Tragekapazitäten von residenten Brut- und Überwinterungspopulationen, frühere Ankunfts- und Durchzugszeiten oder Änderungen im Verbreitungsgebiet. Extremereignisse wie Starkregen, Orkane und Dürren treten aufgrund des Klimawandels in zunehmendem Maße auf und richten erhebliche Schäden an. Durch den Anstieg des Meeresspiegels drohen einige Inselstaaten ganz zu verschwinden. Diese Veränderungen sind von globaler Bedeutung und stellen eine der größten - auch international - Herausforderungen für Politik und Gesellschaft und damit auch für das internationale Rechtsgefüge dar.

Das vorliegende Werk ist in zwei getrennten Bänden erschienen, es verfolgt das Ziel, das internationale Klimaschutzrecht im Zusammenhang internationaler Klimapolitik systematisch darzustellen und einer wissenschaftlichen Gesamtbetrachtung und -bewertung zu unterwerfen. An einem solchen Werk fehlt es bislang.

Herausgeber dieses Werks sind Prof. Dr. Oliver Ruppel, Prof. für Völkerrecht an der Universität Stellenbosch, Südafrika, Prof. Dr. Christian Roschmann, Direktor des Rechtsstaatsprogramms der KonradAdenauer-Stiftung für Subsahara Afrika und Prof. an der Hochschule Harz und Dr. Dr. Katharina Ruppel-Schlichting, Direktorin des Legal Research and Development Trust for Africa, Windhoek Namibia und Stellenbosch, Südafrika.

Untersucht werden von den Autoren in 60 Beiträgen insbesondere die Rechte und Pflichten von Staaten und internationalen Akteuren und deren Zusammenarbeit. Die fachübergreifenden Beiträge werden von 55 internationalen Experten erörtert.

Der erste Band befasst sich in sechs Kapiteln mit internationalen Rechtsfragen und den zugrunde liegenden Rechtsinstrumenten, welche den Klimawandel betreffen.

Kapitel 1 hat das Generalthema: Internationales Klimawandelrecht - eine neue Rechtsdisziplin. In dem ersten Aufsatz untersuch Ruppel die Schnittpunkte zwischen dem Recht und dem Klimawandel. Diese Diskussion zeigt auch den interdisziplinären Zugang zum

Ass. jur. Jochen Schumacher,

Institut für Naturschutz und Naturschutzrecht Tübingen,

Tübingen, Deutschland
Klimawandel auf und ist insbesondere wichtig für die Abgrenzung für ein Klimawandelrecht. Der Beitrag von Koch zu dem Thema „Klimawandelrecht: Zielvorstellungen, Instrumente und Strukturen eines neuen Rechtsgebiets", stellt die Klimapolitik in Deutschland und der Europäischen Union vor und behandelt u. a. den Rechtsrahmen für die Energieeffizienz und die Förderung erneuerbarer Energien. Kapitel 2 beschäftigt sich mit Fragen des Klimawandels und seinen Auswirkungen auf die Menschenrechte. Beleuchtet werden dabei die vielfältigen Aspekte wie z. B. ökonomische, soziale und kulturelle Rechte die durch den Klimawandel ebenso beeinträchtigt werden können. In Kapitel 3 werden Fragen des Klimawandels in Bezug auf den Handel und des Investitionsrecht beleuchtet. So setzt sich z.B. Ghaleieh kritisch mit der europäischen Klimapolitik und insbesondere mit dem Emissionshandelssystem auseinander. Kapitel 4 ist dem Seerecht und dem zu erwartenden Anstieg des Meeresspiegels durch den Klimawandel gewidmet. So befassen sich Wannier und Gerrard mit dem Problem, dass Staaten durch Anhebung des Meeresspiegels in ihrer staatlichen Existenz und Souveränität gefährdet werden können. Kapitel 5 hat als Thema: Fragen der gerichtlichen Kontrolle und völkerrechtliche Versuche eine solche Kontrollmöglichkeit auf staatlicher Seite zu etablieren. Kemp befasst sich hier in seinem Aufsatz mit der Frage, wie die internationale Strafgerichtsbarkeit für die Durchsetzung des internationalen Klimaschutzes und die Verantwortung für den Klimawandel herangezogen werden kann. Ebert gibt einen Überblick über Rechtsfragen des Klimawandels und der Haftung. Das 6. Kapitel rundet den ersten Band ab und stellt das Klimawandelrecht z. B. in Bezug auf das Risikorecht, das Bergrecht und die Kohlendioxidspeicherung (CCS) dar.

Der zweite Band beleuchtet spezifische Fragestellungen des Klimaschutzes. Ausgehend von dem Rahmenübereinkommen der Vereinten Nationen über Klimaänderungen (UNFCCC) werden rechtliche, politische und transdisziplinäre Fragestellungen des Klimaschutzes im Spannungsgefüge internationaler Diplomatie und globaler Governance untersucht

Das Thema des 1. Kapitels ist die „Globale Klima Governance Entwicklungen und Herausforderungen“. Die Autoren des 2. Kapitels beschäftigen sich mit der Frage der globalen Klimasteuerung und der Diplomatie. Dabei stellt Walker in seinem Aufsatz die globale Herausforderung des Klimawandels und des Seerecht gegenüber. Hanschel unterzieht UNEP plus X einer kritischen Untersuchung. Die Autoren des 3. Kapitels beschäftigen sich mit dem Mix von Klimawandel, Politik und Finanzen. Haibach und Schneider setzen sich in ihrem Aufsatz mit der Frage „Politik des Klimawandels: Rückblick und zukünftige Herausforderungen“ auseinander. Das Thema des 4. Kapitels ist „Klimawandelpolitik, Kooperation und Schutzanstrengungen“. Im Einzelnen beschäftigt sich Ruppel in seinem Beitrag mit Klimawandelrecht und politischen Positionen in der Afrikanischen Union und Entwicklungen in verschiedenen afrikanischen Ländern. Callies und Hey beschäftigen sich in ihrem Beitrag mit der Relevanz der Politik zur Förderung der erneuerbaren Energien in der Europäischen Union. Sie fragen u. a. nach einer etwaigen Verpflichtung der Europäischen Union, durch Planungsmaßnahmen aktiv die Energiepolitik der Mitgliedstaaten zu steuern.

Inhaltlich darf festgehalten werden, dass sich die Beiträge durchgängig auf einem qualitativ hohen Niveau befinden. Dies betrifft sowohl die Grundsätze des internationalen Klimaschutzrechts als auch die verschiedenen einzelnen Perspektiven. Das Werk liefert daher einen wichtigen Beitrag zur Erfassung und Systematisierung des Klimaschutzes als eigenständiges Rechtsgebiet. 